\title{
Muscle dysfunction and exercise limitation in adolescent idiopathic scoliosis
}

\author{
J. Martínez-Llorens*, M. Ramírez ${ }^{\#}$, M.J. Colomina", J. Bagó ${ }^{+}$, A. Molina\#, \\ E. Cáceres ${ }^{\#}$ and J. Gea*
}

ABSTRACT: Adolescent idiopathic scoliosis (AIS) can lead to ventilatory restriction, respiratory muscle weakness and exercise limitation. However, both the causes and the extent of muscle dysfunction remain unclear. The aim of our study is to describe muscle weakness and its relationship to lung function and tolerance to exercise in AIS patients.

Lung and muscle function, together with exercise capacity, were assessed in 60 patients with pronounced spinal deformity $\left(>40^{\circ}\right)$ and in 25 healthy volunteers.

Patients with AIS had only mild to moderate abnormal ventilatory patterns, the most frequent of which were restrictive abnormalities. The function of respiratory and limb muscles and exercise capacity were below normal limits in AIS patients, and were significantly lower than in controls. Exercise capacity was found to correlate with the function of inspiratory, expiratory, upper limb and lower limb muscles which, in addition, were reciprocally interrelated. Multivariate analysis showed that lower limb muscle function is the main contributor to exercise intolerance. There appeared to be no connection between spinal deformity and lung function, muscle function or exercise capacity.

We conclude that AIS patients show generalised muscle dysfunction which contributes to the reduction in their exercise capacity, even in the absence of severe ventilatory impairment.

KEYWORDS: Lung function in disease, respiratory muscle function, skeletal muscle dysfunction, spinal deformity

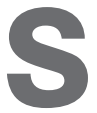
coliosis, defined as a lateral curvature of the spine associated with vertebral rotation, results in chest deformity, back pain, ventilatory restriction, respiratory muscle weakness, exercise limitation and the subsequent impairment of health-related quality of life [1-6]. The severity of the scoliosis is usually determined by the angle between the upper and lower limits of the deformity (Cobb's method) [7]. Scoliosis is usually idiopathic but in some cases it can also be secondary to different neuromuscular, vertebral and connective tissue disorders [1,2]. Depending on the age of the patient at the onset of the condition, idiopathic scoliosis can be classified as infantile (from birth to 3 yrs), juvenile (from 3 to 11 yrs) and adolescent ( $\geqslant 11$ yrs) [1]. The prevalence of scoliosis among adolescents is $\sim 1-3 \%$, and it frequently involves the thoracic spine [2]. Therefore, it is no surprise to find that respiratory impairment is considered the most serious consequence of severe scoliosis as patients frequently develop a progressive reduction in their lung volumes (restrictive ventilatory pattern), inspiratory muscle weakness and reduced exercise capacity, and can even suffer a sudden death episode [2-6, 8-10]. Classical therapy of adolescent idiopathic scoliosis (AIS) includes orthopaedic treatment, relative rest and/or rehabilitation and, in the most severe cases, surgery and/or ventilatory support $[2,11-15]$. The causes of exercise limitation in this disorder have not been fully elucidated, but ventilatory restriction and subsequent cardiovascular deconditioning are the most commonly accepted [10, 16]. Another possible cause is limb muscle dysfunction, which has occasionally been observed in some patients [16]. However, relatively few studies have explored in depth the topography, severity and consequences of skeletal muscle dysfunction in AIS. The purpose of our study is extensively to describe skeletal muscle function and its relationships with lung function, exercise capacity, body composition and spinal deformity in young patients with pronounced thoracic AIS.

\section{METHODS}

\section{Patients and study design}

This case-control study was conducted in accordance with the World Medical Association guidelines for research on humans [17]. It was approved by our institutional Ethics Committee (Comité Ético y de Ensayos Clínicos, IMIM - Hospital del

\section{AFFILIATIONS}

*Servei de Pneumología, URMAR, Hospital del Mar, IMIM, CEXS, Universitat Pompeu Fabra, CIBER de Enfermedades Respiratorias (CIBERES), ISC III,

${ }^{\text {\#}}$ Servei de COT, Hospital del Mar, IMIM, Universitat Autónoma de Barcelona,

'Servei d'Anestesia, and

+Servei de COT, Hospital de la Vall d'Hebron, Universitat Autónoma de Barcelona, Barcelona, Spain.

CORRESPONDENCE

J. Gea

Servei de Pneumología

Hospital del Mar

IMIM

Pg. Marítim 25-27

08003 Barcelona

Spain

E-mail: jgea@imim.es

Received:

Feb 132009

Accepted after revision:

Dec 142009

First published online:

Dec 232009 
Mar, Barcelona, Spain) and all patients and controls gave their written informed consent. The size of the sample was calculated on the basis of a previous pilot study [18], and the case group was made up of 60 consecutive patients with AIS recruited in the Dept of Orthopaedics at our two centres (Hospital del Mar amd Hospital de la Vall d'Hebron; both UAB, Barcelona). All subjects showed pronounced spinal deformity, defined by a thoracic Cobb's angle of $>40^{\circ}$. Individuals with bronchial asthma and other pulmonary, cardiovascular or skeletal muscle problems, and those who had ever undergone spinal surgery were excluded from the study. 25 healthy volunteers of similar age and sex distribution to patients were used as controls. Both patients and volunteers were students with normal patterns of physical activity (as assessed by a standardised questionnaire) but who did not do any sport on a regular basis.

\section{Nutritional and body composition}

Body weight was assessed using a conventional weighing chair (SECA, Berlin, Germany). The body mass index was calculated as the body weight divided by the square of the height predicted by arm span, whereas body composition, including proportions of water, fat-free mass, fat mass and the fat-free mass index (FFMI) was determined using bioelectrical impedance (Bodystat, Isle of Man, UK).

\section{Spinal deformity}

Back deformity was assessed in anteroposterior and lateral spine radiographs by two independent observers. For measurement of the Cobb's angle, two lines were drawn parallel to the end plates of the vertebral bodies at the beginning and at the end of the curve in the anteroposterior projection. Subsequently, third and fourth lines were drawn perpendicular to each of the first two lines to define the Cobb's angle [7].

\section{Lung function}

All individuals performed forced spirometry (Datospir92; Sibel, Barcelona, Spain). In addition, static lung volumes, airway resistance, diffusing capacity of the lung for carbon monoxide (DL,CO) (Masterlab; Jaeger, Würzburg, Germany) and oxygen saturation (ARTEMAR MM205 Oximeter; Medical $\mathrm{AB}$, Stockholm, Sweden) were determined in AIS patients using standard procedures. All lung function variables except oxygen saturation are expressed as \% predicted values for the local population [19-21]. Arm span was again used to calculate the predicted height. Ventilatory and gas exchange abnormalities were defined as follows [22]. Obstructive ventilatory pattern: ratio of forced expiratory volume in $1 \mathrm{~s}$ to forced vital capacity (FEV1/FVC) $<70 \%$. Restrictive ventilatory pattern: FVC and total lung capacity (TLC) $<80 \%$ pred and FEV1/FVC $\geqslant 70 \%$. Mixed ventilatory pattern: similar abnormal percentages but without previous criteria for obstructive or restrictive abnormalities. Gas exchange impairment: oxygen saturation $\leqslant 98 \%$ and/or single-breath $D$ L,CO $<80 \%$ pred.

\section{Respiratory muscle function}

The strength of respiratory muscles was assessed in all individuals by measuring maximal inspiratory and expiratory pressures at the mouth (MIP and MEP, respectively). These pressures were generated during maximal efforts performed against an occluded airway [23]. While MIP was obtained from residual volume, MEP was determined from TLC. The mouthpiece used in the manoeuvres (Sibel) had a small orifice to minimise the participation of face and mouth muscles and was connected to a pressure transducer attached to a digital recorder (BIOPAC; BIOPAC Systems, Schooner, CA, USA). The highest value of three reproducible manoeuvres $(<5 \%$ variability between values) was used for the analysis and reference values were those previously published for a Mediterranean population [24].

The endurance of inspiratory muscles was assessed in all subjects using a threshold loading test as described previously [25]. In short, individuals breathed against incremental threshold loads until exhaustion and the maximal pressure that they could maintain for at least $60 \mathrm{~s}$ was defined as the maximal sustainable pressure $\left(P_{\text {th, }}\right.$ max $)$.

\section{Limb muscle function}

Handgrip (HG) strength was measured in all subjects with a hydraulic dynamometer (JAMAR 5030J1; Sammons Preston, Bolingbrook, IL, USA) attached to the digital recorder. All subjects were seated with their shoulder adducted and neutrally rotated, the elbow flexed at $90^{\circ}$ and the forearm and wrist in a neutral position. Each individual pressed the dynamometer, making at least three manoeuvres with each hand (dominant and non-dominant). The highest value of three valid and reproducible manoeuvres was used in the analysis. Reference values published by MATHIOWETz et al. [26] were used for subjects aged $>18$ yrs, whereas predictions for younger individuals were obtained from our own laboratory database (unpublished data).

In two thirds of the patients and all control subjects the strength of the quadriceps muscle was also assessed using the technique described in a previous study [27]. In short, quadriceps isometric maximum voluntary contraction (QMVC) was obtained through a brief $(3 \mathrm{~s})$ effort performed with one leg and then the other, the patients were seated with both their trunk and thigh fixed on a rigid support of an exercise platform (Domyos HGH 050; Decathlon, Lille, France). The dynamometer was connected to the recording system and the highest value from three reproducible manoeuvres was used for the analysis. Reference values used were those published by BOHANNON [28].

\section{Exercise capacity}

This was determined by a standardised incremental exercise test. In short, subjects pedalled in an electrically braked cycloergometer (Ergoselect 1000L; Ergoline GmbH, Bitz, Germany) and were encouraged to continue until they could no longer sustain the target frequency (45-50 rpm). Loads were increased by $25 \mathrm{~W}$ every $2 \mathrm{~min}$. Different ventilatory, cardiovascular, metabolic and oxygenation variables were monitored throughout the test using a calibrated exercise system (Oxycom Alpha; Jaeger), a standard electrocardiograph (78352A; Hewlett Packard, Bad Homburg, Germany), an automatic sphygmomanometer and a finger probe connected to the aforementioned digital recorder. Normal values published by JONES et al. [29] were used as the reference for physiological parameters, except for the maximum heart rate which was calculated from a standard equation published by WASSERMAN et al. [30]. Finally, dyspnoea and leg discomfort 
TABLE 1 General and nutritional characteristics

\begin{tabular}{|c|c|c|c|}
\hline & AIS patients & Controls & p-value \\
\hline \multicolumn{4}{|l|}{ General characteristics } \\
\hline Subjects & 60 & 25 & \\
\hline Female/male & $50 / 10$ & $20 / 5$ & NS \\
\hline Age yrs & $20 \pm 3$ & $21 \pm 2$ & NS \\
\hline Smoking & $0.5 \pm 0.3(12)$ & $0.5 \pm 0.2(13)$ & NS \\
\hline Thoracic Cobb's angle & $56 \pm 17$ & & \\
\hline Lumbar Cobb's angle & $35 \pm 16$ & & \\
\hline Vertebrae involved & $7 \pm 1$ & & \\
\hline \multicolumn{4}{|l|}{ Anthropometric characteristics } \\
\hline BMI $\mathrm{kg} \cdot \mathrm{m}^{-2}$ & $19.9 \pm 3.2$ & $22.4 \pm 2.9$ & $<0.001$ \\
\hline Weight kg & $56 \pm 10$ & $62 \pm 8$ & $<0.01$ \\
\hline Calculated height from arm span $\mathrm{cm}$ & $167 \pm 9$ & $167 \pm 8$ & NS \\
\hline $\mathrm{FFMI} \mathrm{kg} \cdot \mathrm{m}^{-2}$ & $14.1 \pm 1.1$ & $15.4 \pm 1.6$ & $<0.01$ \\
\hline
\end{tabular}

were measured using Borg scales from 0 (none) to 10 (maximal) at rest and at the end of the exercise test.

\section{Statistical analysis}

Data are presented as mean \pm SD. Since the distribution of different quantitative variables was normal (tested using the Kolmogoroff-Smirnoff test), comparisons between groups were made using the parametric unpaired t-tests, whereas the relationships between different variables in AIS patients were evaluated using the Pearson coefficient. Only hypothesisdriven correlations were tested. Subsequently, different variables were also analysed using a multiple regression model. A $\mathrm{p}$-value of $\leqslant 0.05$ was considered significant.

\section{RESULTS}

\section{General and nutritional characteristics}

The main general data for both groups are shown in table 1. Patients had lower body weight, body mass index and FFMI than controls, and all patients had pronounced spinal deformity.

\section{Lung function}

Although mean values for most lung function variables were within the normal range (table 2), they showed significant impairment compared with those in controls. In addition, as many as half the patients had an abnormal ventilatory pattern (table 3). Pure restrictive ventilatory abnormalities were frequent and only a small percentage of patients had a mixed or pure obstructive pattern. In all but one case, ventilatory abnormalities were only mild to moderate. Gas exchange at rest was only abnormal in a relatively low proportion of the AIS patients, who showed mild reductions in DL,CO and/or oxygen saturation. No significant correlations were found between the severity of the spinal deformity (as assessed by the thoracic Cobb's angle) and different lung function variables.
TABLE 2 Lung function, muscle function and exercise

\begin{tabular}{|c|c|c|c|}
\hline & AIS patients & Controls & p-value \\
\hline \multicolumn{4}{|l|}{ Lung function } \\
\hline $\mathrm{FEV}_{1} / \mathrm{FVC} \%$ & $80 \pm 6$ & $84 \pm 5$ & $<0.05$ \\
\hline FEV $1 \%$ pred & $83 \pm 16$ & $105 \pm 11$ & $<0.001$ \\
\hline FVC \% pred & $86 \pm 14$ & $105 \pm 12$ & $<0.001$ \\
\hline FRC $\%$ pred & $86 \pm 11$ & & \\
\hline TLC \% pred & $89 \pm 17$ & & \\
\hline RV/TLC \% & $30 \pm 9$ & & \\
\hline$D L, C O \%$ pred & $91 \pm 11$ & & \\
\hline $\mathrm{Sp}_{\mathrm{O}} \mathrm{O}_{2}^{\#} \%$ & $99 \pm 0.4$ & $99.6 \pm 0.3$ & NS \\
\hline \multicolumn{4}{|l|}{ Muscle function } \\
\hline MIP $\mathrm{cmH}_{2} \mathrm{O}$ & $85 \pm 26$ & $112 \pm 16$ & $<0.001$ \\
\hline MIP \% pred & $71 \pm 19$ & $95 \pm 15$ & $<0.001$ \\
\hline MEP $\mathrm{cmH}_{2} \mathrm{O}$ & $105 \pm 36$ & $143 \pm 39$ & $<0.001$ \\
\hline MEP \% pred & $69 \pm 19$ & $91 \pm 18$ & $<0.001$ \\
\hline Pth, $\max \mathrm{cmH}_{2} \mathrm{O}$ & $59 \pm 20$ & $97 \pm 22$ & $<0.001$ \\
\hline HG dominant kg & $27 \pm 7$ & $32 \pm 7$ & $<0.001$ \\
\hline HG dominant \% pred & $78 \pm 14$ & $91 \pm 13$ & $<0.01$ \\
\hline HG non-dominant kg & $25 \pm 7$ & $30 \pm 8$ & $<0.001$ \\
\hline HG non-dominant \% pred & $79 \pm 17$ & $95 \pm 14$ & $<0.01$ \\
\hline QMVC dominant kg & $38 \pm 11$ & $46 \pm 24$ & $<0.01$ \\
\hline QMVC dominant \% pred & $71 \pm 22$ & $95 \pm 28$ & $<0.01$ \\
\hline QMVC non-dominant kg & $36 \pm 12$ & $44 \pm 21$ & $<0.01$ \\
\hline QMVC non-dominant \% pred & $73 \pm 20$ & $94 \pm 24$ & $<0.01$ \\
\hline \multicolumn{4}{|l|}{ Exercise } \\
\hline WRmax \% pred & $58 \pm 15$ & $97 \pm 9$ & $<0.001$ \\
\hline$V^{\prime} \mathrm{O}_{2}$ max $\%$ pred & $60 \pm 16$ & $94 \pm 11$ & $<0.001$ \\
\hline$V^{\prime}$ Emax \% pred & $54 \pm 19$ & $67 \pm 8$ & NS \\
\hline $\mathrm{RR} \mathrm{min}^{-1}$ & $43 \pm 8(+126)$ & $34 \pm 7(+88)$ & $<0.01$ \\
\hline HRmax \% pred & $79 \pm 9$ & $97 \pm 8$ & $<0.001$ \\
\hline $\mathrm{Sp}, \mathrm{O}_{2}$ at peak exercise $\%$ & $98.3 \pm 0.9(-0.5)$ & $99.2 \pm 0.7$ & NS \\
\hline
\end{tabular}

Data are presented as mean $\pm \mathrm{SD}$, unless otherwise stated. Data for respiratory rate (RR) and arterial oxygen saturation measured by pulse oximetry $\left(\mathrm{Sp}, \mathrm{O}_{2}\right)$ are presented as mean \pm SD (\% $\Delta$ from baselines). AIS: adolescent idiopathic scoliosis; FEV1: forced expiratory volume in $1 \mathrm{~s}$; FVC: forced vital capacity; \% pred: percentage of predicted; FRC: functional residual capacity; TLC: total lung capacity; RV: residual volume; $\mathrm{LL}, \mathrm{CO}$ : diffusing capacity of the lung for carbon monoxide; MIP: maximal inspiratory pressure; MEP: maximal expiratory pressure; Pth,max: maximal sustainable inspiratory pressure; HG: handgrip: QMVC: quadriceps maximum isometric voluntary contraction; WRmax: maximum work rate; $V^{\prime} \mathrm{O}_{2}$,max: maximum oxygen uptake; $V^{\prime} E$,max: maximum minute ventilation; HRmax: maximum heart rate; NS: nonsignificant. ${ }^{\#}$ : inspiratory oxygen fraction 0.21

TABLE 3 Ventilatory and gas exchange impairment observed in patients with adolescent idiopathic scoliosis

Normal ventilatory pattern

$29(48)$

Restrictive pattern

$23(38)$

Obstructive pattern

4 (7)

Mixed pattern

$4(7)$

Abnormal gas exchange

$9(15)$

Data are presented as n (\%). 

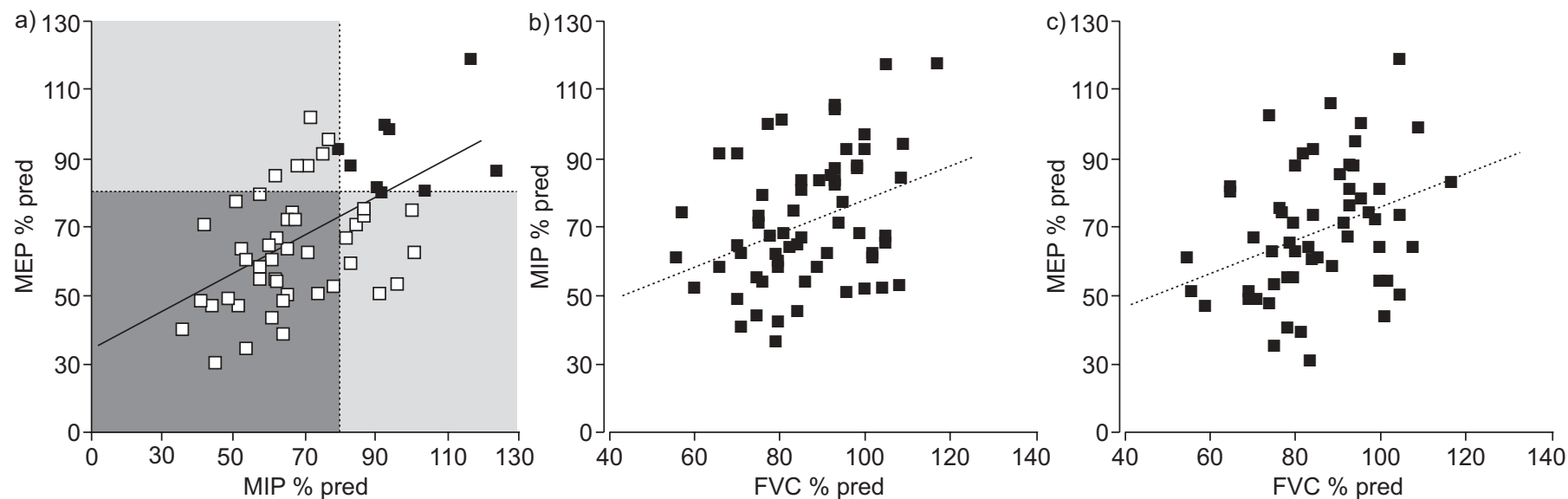

FIGURE 1. a) Relationships between \% predicted (\% pred) maximal inspiratory pressure (MIP) and maximal expiratory pressure (MEP). 1 : impairment in either MIP or MEP; E: patients with both MIP and MEP muscle dysfunction. $r=0.566 ; p<0.001$. b) Relationship between lung function (represented by forced vital capacity; FVC) and strength 0 inspiratory muscles (MIP). $r=0.354 ; p<0.01$. c) Relationship between lung function (represented by FVC) and strength of expiratory muscles (MEP). $r=0.356 ; p<0.01$.

\section{Respiratory muscle function}

Most of the patients with AIS (83\%) had respiratory muscle dysfunction characterised by reduced MIP, MEP and/or $P$ th,max (table 2 and fig. 1a). In addition, all these variables were significantly lower in patients than in controls. Interestingly, a direct relationship was found between respiratory muscle strength (expressed by either MIP or MEP) and lung function impairment (as represented by FVC) (fig. 1b and c). However, we did not detect any significant correlations between respiratory muscle function and the severity of the spinal deformity.

\section{Limb muscle function}

The strength of the limb muscles was reduced in more than half of the patients (53\% for the non-dominant HG and $61 \%$ for the non-dominant quadriceps muscle), with the values being lower than those obtained in control subjects (table 2). No differences were observed between data obtained on the dominant and non-dominant sides for either the hands or the legs. Interestingly, the strength of both non-dominant HG and quadriceps muscles directly correlated with that of respiratory muscles: MIP: $\mathrm{r}=0.338, \mathrm{p}<0.05$ and $\mathrm{r}=0.568, \mathrm{p}<0.001$; MEP: $\mathrm{r}=0.278, \mathrm{p}=0.05$ and $\mathrm{r}=0.455, \mathrm{p}<0.01$, respectively. Moreover, the strength of both the non-dominant $\mathrm{HG}$ and the quadriceps muscle was directly related to FFMI $(r=0.667$ and 0.598 , respectively, $\mathrm{p}<0.01$ for both).

\section{Exercise capacity}

Data obtained in the exercise test are shown in table 2. Most of the patients $(91 \%)$ had a mild to severe reduction in exercise capacity, with the limiting symptom being leg discomfort in all cases. Moreover, exercise capacity (represented by the maximum work rate; WRmax) correlated positively with the strength of both the respiratory and peripheral muscles (fig. 2) but, once again, no significant relationship was observed with either the severity of the spinal deformity or with lung function. Multivariate analysis using \% predicted values did not add any further information since only MEP
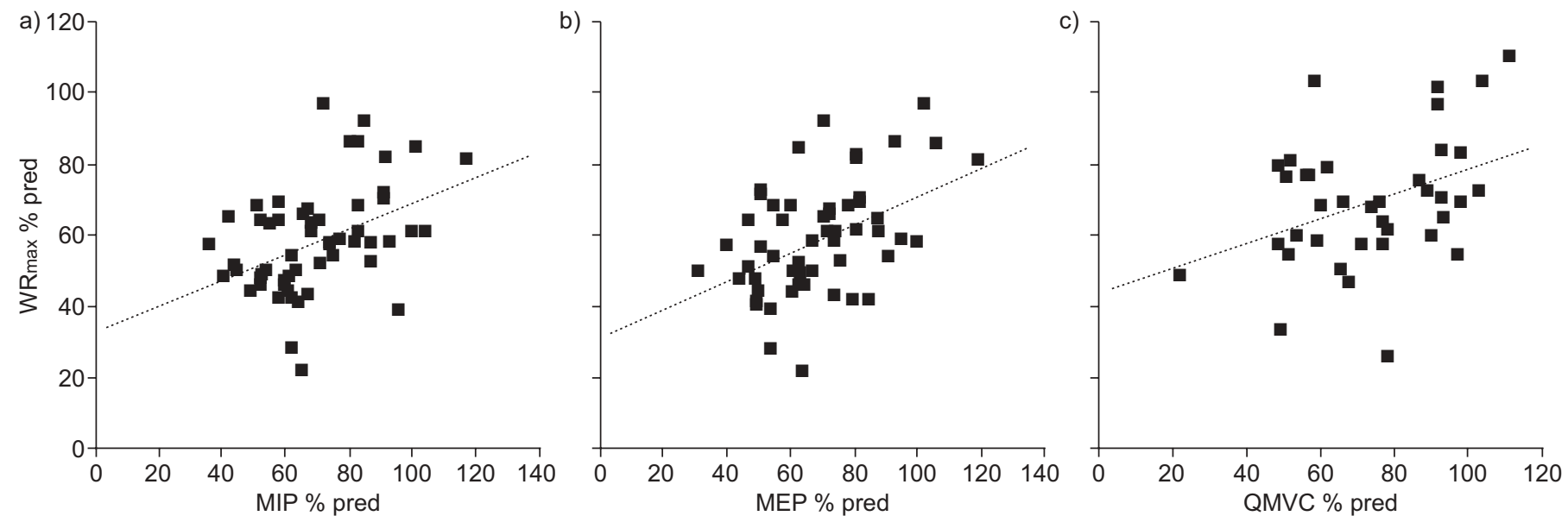

FIGURE 2. Relationship between exercise capacity (\% predicted (\% pred) maximum work rate (WRmax)) and the strength of a) inspiratory muscles (maximal inspiratory pressure; MIP), b) expiratory muscles (maximal expiratory pressure; MEP) and c) limb muscles (quadriceps isometric force; QMVC). a) $r=0.427, p<0.001 ; b) r=0.477$, $p<0.001 ; c) r=0.389, p=0.014$. 
reached a significant value for the prediction of exercise capacity. However, since prediction equations for peripheral muscle strength had not been validated to the same degree as those corresponding to lung function, respiratory muscle force or exercise, we also performed this multivariate analysis using actual values. This approach clearly identified the function of peripheral muscles as the main factor contributing to exercise capacity $\left(R^{2}=0.623, p<0.01\right.$ for the overall analysis; $p=0.02$ for lower limb strength and $\mathrm{p}=0.05$ for $\mathrm{HG}$; in this case, the strength of the expiratory muscles did not reach statistical significance, $\mathrm{p}=0.08)$. Moreover, the prediction of the WRmax turns out to be a function of the following equation:

$$
\begin{aligned}
& \mathrm{WR} \max =0.335(\mathrm{MEP})+1.435(\mathrm{HG})+1.244(\mathrm{QMVC})-0.175 \\
& \text { (MIP) - } 3.629
\end{aligned}
$$

where both HG and QMVC were those obtained in the nondominant limbs.

\section{DISCUSSION}

The present study demonstrates that young patients with AIS with pronounced spinal deformity have generalised skeletal muscle weakness and exercise limitation, even in the absence of major ventilatory defects. Moreover, muscle function was the main predictor of exercise limitation and appeared not to be markedly influenced by the degree of the spinal deformity. These novel results reveal the central role played by muscle dysfunction in clinical manifestations of AIS and strongly suggest that this abnormality is probably the consequence of systemic rather than local factors.

\section{Lung function}

In keeping with most of the previous reports, ventilatory abnormalities were very common $(52 \%)$ and respiratory restriction was the most frequent feature found in our patients $[8,31,32]$. However, functional impairment was only mild to moderate in most cases and did not correlate with the level of thoracic deformity. Most surprisingly, obstructive abnormalities were also present in a non-negligible number of individuals ( $14 \%$ if both pure obstructive and mixed ventilatory patterns are taken into account). This has also been occasionally reported in AIS [33, 34], but is thought to be less prevalent and might be related to the possible influence of chest deformity and/or respiratory muscle dysfunction on the cross-sectional area of the airways.

Many studies have already investigated lung function in patients with scoliosis, exploring, in particular, the relationships between the chest deformity and ventilatory impairment $[5,8,9,35-37]$. Some of these reports have concluded that the former is the main cause of the latter $[8,9]$. Other authors, by contrast, obtained similar results to those found in the present study and have been unable to establish a direct link between the severity of spinal deformity and lung function [35-37]. These discrepancies can be explained by the heterogeneity of the populations included in the different studies, where idiopathic scoliosis was frequently mixed with secondary scoliosis, and clinical subtypes, severity of chest deformities and smoking histories were very diverse. To avoid any confusion that might arise from such factors, we only included patients with clinical AIS and severe thoracic deformity, with no relevant smoking history or associated respiratory diseases.
Another potential technical limitation of many of the previous studies is the extensive use of the actual height of the patients, which can be significantly decreased in scoliosis, for calculations of predicted values. This could have contributed to the overestimation of patients' functional data and the subsequent underestimation of their respiratory impairment. To avoid this limitation, we used arm span to calculate the theoretical height of the patients [38]. One plausible explanation for the fact that neither our study nor some of the previous studies have detected any relationship between thoracic deformity and lung function may be the contribution of respiratory muscle impairment to the values obtained in the lung function assessment. It is a well-attested fact that impairment of respiratory muscle function can result in or contribute to different ventilatory abnormalities in respiratory and neuromuscular disorders. This is also suggested by the relationship observed in our study between maximal respiratory pressures and FVC.

\section{Muscle dysfunction}

Loss of muscle function occurred frequently in our patients and affected all the different muscle groups explored in the present study: inspiratory muscles, expiratory muscles, upper limbs and lower limbs, as well as dominant and non-dominant extremities. Some previous studies have assessed muscle function in AIS patients. However, most of these reports confined themselves to the determination of respiratory muscle strength, which was found to be impaired to variable degrees $[9,34]$. To the best of our knowledge, only KEARON et al. [16] explored different muscle territories, reporting that the strength of both respiratory and limb muscles was reduced in AIS patients. This was associated with a reduction in the calculated lean mass, suggesting a role for nutritional abnormalities and/or muscle deconditioning. Our study serves to confirm and extend these previous findings. This is the first study to explore body composition along with muscle function, as well as assessing respiratory muscle endurance in AIS patients. It shows that, as suggested by indirect calculations performed by KEARON et al. [16], fat-free mass is decreased in such individuals. Moreover, this reduction was proportional to that of limb muscle strength. This suggests that the former should have contributed to the latter in our patients. In addition, the current study demonstrates that respiratory muscle endurance is also impaired in AIS patients. Endurance is a very important functional property which is probably even more relevant than maximal strength for daily life activities. It is less dependent than strength on muscle mass, and is more related to the aerobic capacity of the muscle. Unfortunately, the assessment of muscle endurance is relatively difficult [27, 39] and there is also a lack of reference values, both of which might explain the absence of previous reports.

The causes of muscle dysfunction in AIS remain unclear, but our results suggest a relevant role for systemic mechanisms. Several different factors can influence muscle function. Some of them, such as the nutritional status, the level of physical activity or systemic inflammation, can be shared by different muscles, while others are specific for each muscle group. Inspiratory muscles, for instance, are probably subject to increased mechanical loads secondary to the spinal deformity and the ventilatory defects present in AIS patients. In addition, 
their length-force relationships can be altered as a consequence of chest deformity. Expiratory muscles might also be subject to increased mechanical loads in AIS. However, the impact of geometrical distortion should be less important in this muscle group, the main representatives of which are in the abdominal wall. In this context of mechanical difficulties, it is not surprising that MIP and MEP were reduced in our patients. However, the patients also had peripheral muscle dysfunction, and it is unlikely that this could also be a direct result of changes occurring in the thorax. Impairment of limb muscle function must be explained by other factors. Moreover, the fact that muscle function impairment was generalised and proportional across different muscle groups in our patients, along with a corresponding reduction in FFMI, reinforces the hypothesis that systemic factors also have a relevant role in muscle dysfunction associated with AIS. The loss of muscle mass is one well-known cause of muscle dysfunction, and it can partially explain the loss of limb strength in our patients. A second possibility, which can also contribute to the loss of muscle mass, is muscle deconditioning. This factor can act on muscle status both directly and indirectly through a decrease in the anabolic stimuli that the action of a given muscle causes in the others. However, there are several arguments against a relevant role for muscle deconditioning in our patients. First, muscle impairment involved both dominant and nondominant hands to a similar extent. The muscles of the dominant hand might be less affected by deconditioning than the contralateral ones, since self-care activities probably persist even in an advanced sedentary state and the rate of decline in strength with age is slower in the dominant than in the nondominant upper limb [40]. In addition, involuntary deconditioning was very unlikely in our young patients since their ventilatory defect was too mild to induce significant changes in their lifestyle. However, we cannot rule out a voluntary reduction in the level of their physical activity. In fact, this recommendation has been common for a long time in the management of AIS [41, 42] and, in any case, patients with marked scoliosis will almost certainly avoid situations such as sport or outdoor activities where their deformities would be more evident. However, our patients reported a normal level of ordinary activities and, in accordance with more recent evidence $[43,44]$, the most extensive clinical practice in our area is to recommend exercise. Muscle deconditioning might therefore have been present, but our results suggest the presence of additional factors. These might include hormonerelated and inflammatory phenomena. On the one hand, female patients with AIS frequently show hypo-oestrogenic manifestations which can include reduced bone density and decreased muscle mass [45]. On the other hand, low nocturnal melatonin levels have occasionally been reported in some subgroups of patients with scoliosis such as those with rapid progression [46]. Melatonin might play a relevant role in the preservation of muscle mass under a variety of conditions by inhibiting cytokine release [47]. Unfortunately, determination of oestrogen and melatonin levels was not included in our study. Finally, a very recent report suggests that oxidative stress and local inflammation are present in the peripheral muscles of adult patients with chest restriction [48], and this fact has been shown to contribute to muscle dysfunction in other entities such, as chronic obstructive pulmonary disease or sepsis [49].

\section{Exercise capacity}

Most of the patients included in our study had significant exercise limitation. Moreover, variables related to muscle function were the main predictors of exercise capacity in our patients. Although reductions in exercise capacity have been observed previously in patients with AIS [10, 16], our results are impressive. Most authors of previous studies believe that ventilatory and cardiac limitations are the main causes of impairment in exercise capacity among AIS patients [10, 16]. However, this hypothesis is not supported by our data, where ventilatory reserve at peak exercise was relatively high (46\%), no changes were observed in blood oxygenation and all patients stopped exercising due to leg symptoms. Moreover, we found a significant relationship between exercise capacity and function in different muscle groups. KEARON et al. [16] reported that exercise potential was not related either to the severity of the spinal deformity or to the ventilatory defect, but rather to inspiratory muscle function. Although a cause-effect relationship cannot be established within the parameters of the present study, our results strongly suggest a relevant role for muscle dysfunction in exercise limitation shown by AIS patients. However, we cannot entirely rule out a role for cardiovascular or ventilatory factors in specific AIS subpopulations. Moreover, discrepancies with some of the previous studies are probably related to the different stages of the disease. At the relatively early phase of AIS presented by our patients, muscle dysfunction appears to be predominant. However, as the disease progresses, lung function and cardiac impairment due to chest deformity will be more decisive in limiting the exercise capacity. A progressive restrictive ventilatory pattern will appear, ventilation-perfusion mismatching and subsequent hypoxaemia/hypercapnia will develop and even cor pulmonale will occur. All of these factors contribute to exercise limitation.

\section{Muscle dysfunction and aetiology of AIS}

By definition, AIS is an entity of unknown aetiology. However, different authors have suggested that a dysfunction of muscle tone might occur in individuals with a genetic predisposition who would develop an abnormally flexible spine during the relatively long growth phase $[45,50]$. In other words, according to this theory, muscle dysfunction might be the cause and not the consequence of AIS. In addition, there is some evidence of the presence of abnormalities in the neural mechanisms involved in the control of posture in AIS patients [51]. Moreover, structural studies of paraspinal muscles have shown changes in the proportion of different fibre types [52-54], myofilament disarrays (a sign of muscle damage) and shortened sarcomerae $[55,56]$. In some cases, respiratory and leg muscles were also found to be structurally and/or functionally abnormal $[55,57]$. In keeping with all these findings, our results can also be interpreted as the expression of a primary and systemic muscle disorder which might have led to both spinal deformity and generalised muscle dysfunction. However, more studies are needed to prove such a challenging hypothesis.

\section{Potential limitations of the study}

One of the potential limitations of the present study is that only two thirds of the patients were assessed for leg muscle strength. In fact, the measurement of quadriceps force was incorporated when the pilot phase was concluded and upper limb muscle 
dysfunction was systematically detected. However, there are still a significant number of subjects with this functional evaluation, and data are very consistent with those obtained in the other muscle territories.

Another potential problem, as previously mentioned, is the possible unreliability of the predicted values for young individuals in many of the tests included in the study. To prevent this, we chose equations which were obtained in populations of the same or similar age groups as that of the patients and also included a healthy control population of similar age and sex distribution. This, in addition, provides us with a benchmark for tests in which no reference values are available.

\section{Conclusions}

Young patients with AIS have generalised muscle dysfunction which might have an important role in their exercise limitation, and which occurs even in the absence of advanced ventilatory impairment. The causes of this muscle function impairment still remain obscure, but our data strongly suggest that they are related to systemic factors. Our results add new information to the traditional view of AIS as a spinal disorder which only affects the physical performance of patients when a severe ventilatory defect is present. Further understanding of the causes and systemic consequences of AIS will enable clinicians to recommend more effective therapies.

\section{SUPPORT STATEMENT}

J. Martínez-Llorens was funded by grants from Fondo de Invstigacions Sanitarias Proyecto de Investigación 070194, Sociedad Española de Neumología y Cirugía Torácica (SEPAR), Societat Catalana de Pneumologia (SOCAP) and the Institut Municipal d'Investigació Mèdica (Ajuts per Residents (Research Fellowhsips for Residents) 2002 and 2003). The study was funded in part by CIBERES (ISC III) and FIS PI 07-0194, Spain.

\section{STATEMENT OF INTEREST}

None declared.

\section{ACKNOWLEDGEMENTS}

The authors would like to thank E. Barreiro for her review of the manuscript, R. Marshall for his help with editing and A. Roig and N. Soler for their technical assistance in the lung function laboratory (all Hospital del Mar, IMIM, Barcelona, Spain).

\section{REFERENCES}

1 Reamy BV, Slakey JB. Adolescent idiopathic scoliosis: review and current concepts. Am Fam Phys 2001; 64: 111-116.

2 Weinstein SL, Dolan LA, Cheng JC, et al. Adolescent idiopathic scoliosis. Lancet 2008; 371: 1527-1537.

3 Laghi F, Tobin MJ. Disorders of the respiratory muscles. Am J Respir Crit Care Med 2003; 168: 10-48.

4 Kim YJ, Lenke LG, Bridwell KH, et al. Prospective pulmonary function comparison following posterior segmental spinal instrumentation and fusion of adolescent idiopathic scoliosis: is there a relationship between major thoracic curve correction and pulmonary function test improvement? Spine 2007; 32: 2685-2693.

5 Takahashi S, Suzuki N, Asazuma T, et al. Factors of thoracic cage deformity that affect pulmonary function in adolescent idiopathic thoracic scoliosis. Spine 2007; 32: 106-112.

6 Shelton YA. Scoliosis and kyphosis in adolescents: diagnosis and management. Adolesc Med State Art Rev 2007; 18: 121-139.
7 Cobb JR. Outline for the study of scoliosis. American Academy of Orthopaedic Surgeons. Instr Course Lect 1948; 5: 61-75.

8 Kearon C, Viviani GR, Kirkley A, et al. Factors determining pulmonary function in adolescent idiopathic thoracic scoliosis. Am Rev Respir Dis 1993; 148: 288-294.

9 Lisboa C, Moreno R, Fava M, et al. Inspiratory muscle function in patients with severe kyphoscoliosis. Am Rev Respir Dis 1985; 132: 48-52.

10 Barrios C, Pérez-Encinas C, Maruenda JI, et al. Significant ventilatory functional restriction in adolescents with mild or moderate scoliosis during maximal exercise tolerance test. Spine 2005; 30: 1610-1615.

11 Upadhyay SS, Mullaji AB, Luk KD, et al. Relation of spinal and thoracic cage deformities and their flexibilities with altered pulmonary function in adolescent idiopathic scoliosis. Spine 1995; 20: 2415-2420.

12 Estenne M, Derom E, De Troyer A. Neck and abdominal muscle activity in patients with severe idiopathic scoliosis. Am J Respir Crit Care Med 1998; 158: 452-457.

13 Parent S, Newton PO, Wenger DR. Adolescent idiopathic scoliosis: etiology, anatomy, natural history and bracing. Instr Course Lect 2005; 54: 529-536.

14 Weiss HR, Negrini S, Rigo M, et al. Indications for conservative management of scoliosis (SOSORT guidelines). Stud Health Technol Inform 2008; 135: 164-170.

15 Dickson JH, Mirkovic S, Noble PC, et al. Results of operative treatment of idiopathic scoliosis in adults. J Bone Joint Surg Am 1995; 77: 513-523.

16 Kearon C, Viviani GR, Kirkley A, et al. Factors influencing work capacity in adolescent idiopathic thoracic scoliosis. Am Rev Respir Dis 1993; 148: 295-303.

17 World Medical Association General Assembly, World Medical Association Declaration of Helsinki: ethical principles for medical research involving human subjects. I Int Bioethique 2004; 15: 124-129.

18 Martínez-Llorens JM, Colomina MJ, Ramírez M, et al. Impact of respiratory and limb muscle dysfunction in exercise tolerance of young patients with idiopathic scoliosis. Eur Respir J 2005; 26: Suppl. 49, 686s-687s.

19 Roca J, Burgos F, Sunyer J, et al. References values for forced spirometry. Eur Respir J 1998; 11: 1354-1362.

20 Roca J, Burgos F, Barbera JA, et al. Prediction equations for plethismografics lung volumes. Respir Med 1998; 92: 454-460.

21 Roca J, Rodriguez-Roisin R, Cobo E, et al. Single-breath carbon monoxide diffusing capacity prediction equations from a Mediterranean population. Am Rev Respir Dis 1990; 141: 1026-1032.

22 Gea J, Rodriguez-Roisin R, Torres A, et al. Lung function changes following Legionnaires' disease. Eur Respir J 1988; 1: 109-114.

23 Black LF, Hyatt RE. Maximal respiratory pressures: normal values and relationship to age and sex. Am Rev Respir Dis 1969; 99: 696-702.

24 Morales P, Sanchis J, Cordero PJ, et al. Maximum static respiratory pressures in adults. The reference values for a Mediterranean Caucasian population. Arch Bronconeumol 1997; 33: 213-219.

25 Ramirez-Sarmiento A, Orozco-Levi M, Guell R, et al. Inspiratory muscle training in patients with chronic obstructive pulmonary disease: structural adaptation and physiologic outcomes. Am J Respir Crit Care Med 2002; 166: 1491-1497.

26 Mathiowetz V, Dove M, Kashman N, et al. Grip and pinch strength: normative data for adults. Arch Phys Med Rehabil 1985; 66 69-72.

27 Coronell C, Orozco-Levi M, Méndez R, et al. Relevance of assessing quadriceps endurance in patients with COPD. Eur Respir J 2004; 24: 129-136.

28 Bohannon RW. Reference values for extremity muscle strength obtained by hand-held dynamometry from adults aged 20 to 79 years. Arch Phys Med Rehabil 1997; 78: 26-32.

29 Jones NL, ed. Clinical Exercise Testing. 3rd Edn. Philadelphia, W.B. Saunders, 1988. 
30 Wasserman K, Hansen JE, Sue DY, et al., eds. Principles of Exercise Testing and Interpretation. 3rd Edn. Philadelphia, Lippincott Williams and Wilkins, 1999.

31 Koumbourlis AC. Scoliosis and the respiratory system. Paediatr Respir Rev 2006; 7: 152-160.

32 Smyth RJ, Chapman KR, Wrigth TA, et al. Pulmonary function in adolescents with mild idiopathic scoliosis. Thorax 1984; 39: 901-904.

33 Alotaibi S, Harder J, Spier S. Bronchial obstruction secondary to idiopathic scoliosis in a child: a case report. J Med Case Reports 2008; 2: 171

34 Boyer J, Amin N, Taddonio R, et al. Evidence of airway obstruction in children with idiopathic scoliosis. Chest 1996; 109: 1532-1535.

35 Upadhyay SS, Mullaji AB, Luk KD, et al. Evaluation of deformities and pulmonary function in adolescent idiopathic right thoracic scoliosis. Eur Spine J 1995; 4: 274-279.

36 Giordano A, Fuso L, Galli M, et al. Evaluation of pulmonary ventilation and diaphragmatic movement in idiopathic scoliosis using radioaerosol ventilation scintigraphy. Nuclear Med Com 1997; 18: 105-111.

37 Newton PO, Faro FD, Gollogly S, et al. Results of preoperative pulmonary function testing of adolescents with idiopathic scoliosis. A study of six hundred and thirty-one patients. J Bone Joint Surg Am 2005; 87: 1937-1946.

38 Hepper NNG, Black LF, Flower WS. Relationship of lung volumes to height and arm span in normal subjects and in patients with spinal deformity. Am Rev Respir Dis 1965; 91: 365-362.

39 Ramirez-Sarmiento A, Orozco-Levi M, Barreiro E, et al. Expiratory muscle endurance in chronic obstructive pulmonary disease. Thorax 2002; 57: 132-136.

40 Luna-Heredia E, Martín-Peña G, Ruiz-Galiana J. Handgrip dynamometry in healthy adults. Clin Nutr 2005; 24: 250-258.

41 Winter RB. Adolescent idiopathic scoliosis. N Engl J Med 1986; 314: 1379-1380.

42 Stone B, Beekman C, Hall V, et al. The effect of an exercise program on change in curve in adolescents with minimal idiopathic scoliosis: a preliminary study. Phys Ther 1979; 59: 759-763.

43 dos Santos L, Stirbulov R, Avanzi O. Impact of physical rehabilitation program on the respiratory function of adolescents with idiopathic scoliosis. Chest 2006; 130: 500-505.

44 Negrini S, Zaina F, Romano M, et al. Specific exercises reduce brace prescription in adolescent idiopathic scoliosis: a prospective controlled cohort study with worst-case analysis. J Rehabil Med 2008; 40: 451-455.

45 Warren MP, Brooks-Gunn J, Hamilton LH, et al. Scoliosis and fractures in young ballet dancers. N Engl J Med 1986; 314: 1348-1353.

46 Machida M, Dubousset J, Imamura Y, et al. Melatonin. A possible role in pathogenesis of adolescent idiopathic scoliosis. Spine 1996; 21: 1147-1152.

47 Mantovani G, Macciò A, Massa E, et al. Managing cancer-related anorexia/cachexia. Drugs 2001; 61: 499-514.

48 Swallow EB, Barreiro E, Gosker H, et al. Quadriceps muscle strength in extra-pulmonary restrictive lung disease. Eur Respir $J$ 2009; 34: 1429-1435.

49 Gea J, Barreiro E, Orozco-Levi M. Skeletal muscle adaptation to disease states. In: Bottinelli R, Reggiani C, eds. Skeletal Muscle Plasticity in Health and Disease: From Genes to Whole Muscle. Doordrecht, Springer, 2006; pp. 315-360.

50 Lowe TG, Edgar M, Margulies JY, et al. Etiology of idiopathic scoliosis: current trends in research. J Bone Joint Surg Am 2000; 82A: 1157-1168.

51 Herman R, Mixon J, Fisher A, et al. Idiopathic scoliosis and the central nervous system: a motor control problem. The Harrington lecture, 1983. Scoliosis Research Society. Spine 1985; 10: 1-14.

52 Bylund P, Jansson E, Dahlberg E, et al. Muscle fiber types in thoracic erector spinae muscles. Fiber types in idiopathic and other forms of scoliosis. Clin Orthop Relat Res 1987; 214: 222-228.

53 Meier MP, Klein MP, Krebs D, et al. Fiber transformations in multifidus muscle of young patients with idiopathic scoliosis. Spine 1997; 22: 2357-2364.

54 Mannion AF, Meier M, Grob D, et al. Paraspinal muscle fibre type alterations associated with scoliosis: an old problem revisited with new evidence. Eur Spine J 1998; 7: 289-293.

55 Sahgal V, Shah A, Flanagan N, et al. Morphologic and morphometric studies of muscle in idiopathic scoliosis. Acta Orthop Scand 1983; 54: 242-251.

56 Low WD, Chew EC, Kung LS, et al. Ultrastructures of nerve fibers and muscle spindles in adolescent idiopathic scoliosis. Clin Orthop Relat Res 1983; 174: 217-221.

57 Trontelj JV, Fernandez JM. Single fibre EMG in juvenile idiopathic scoliosis. Muscle Nerve 1988; 11: 297-300. 